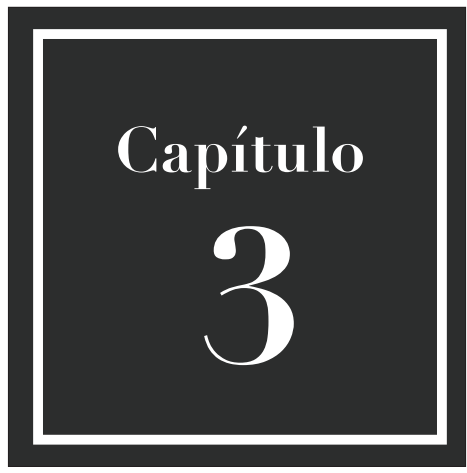

\title{
FUNDAMENTACIÓN FILOSÓFICA Y EPISTEMOLÓGICA
}

Para reforzar lo planteado en el capítulo anterior, se hace necesario el conocimiento de las principales posturas filosóficas y epistemológicas que ayudan a orientar parámetros de acción, métodos, objetivos, intereses y expectativas, por cuanto todo resultado adecuado de la indagación científica contribuye a consolidar algunas posturas ideológicas y epistémicas con la generación de la teoría respectiva que tienen relación por la posición que se asuma ontológicamente ante la correlación entre el ser y el pensar, lo neutral y lo relativo, lo material y lo espiritual (Guadarrama, 2009).

Por su parte, Padrón (2007), planteó que se manejan dos variables para establecer las orientaciones epistemológicas, como la gnoseológica, concerniente a las opiniones acerca del principio del conocimiento, resumida en dos valores: empirismo-racionalismo, y la otra es de tipo ontológico, relacionada con las evidencias de las interacciones sujeto/realidad, idealismo/realismo. De acuerdo con esto, se tiene la realidad de los humedales urbanos aunado al conocimiento con sustento teórico de esa realidad, siguiendo métodos que apuntan a la creación de un conocimiento de propósito y de transformación, por medio de la discusión, la deliberación y la obra combinada de saberes entre los diferentes representantes de un territorio para lograr la innovación social.

En este proceso se involucran e interactúan la teoría y la práctica, el saber técnico y el cotidiano, donde la población o comunidad sea la autogestora del proceso, cuya finalidad se orienta hacia la transformación social, por medio de reflexión, investigación continua y análisis crítico de la realidad (Lewin, 1947). De acuerdo con lo mencionado, la estrategia en la gestión ambiental participativa, en las últimas décadas se ha posicionado a nivel mundial como una alternativa de modelo de investigación social que trata de involucrar a todos los actores participantes de los fenómenos sociales en la construcción de alternativas retroalimentadas para la solución de conflictos.

En otro orden de ideas, la integración de la población cuya realidad se aborda en la construcción de procesos de conocimiento y actuación sobre esta realidad, facilita el análisis y la comprensión de los problemas, necesidades, capacidades y recursos de la población, y permite aplicar acciones para su solución, a la vez que empodera su movilización en torno a ellos. 
Como resultado de esto, se generaría un conocimiento a partir del propio conocimiento y experiencia popular, que conectados con otros procesos similares daría lugar al señalado empoderamiento o incremento del poder político de las comunidades.

De tal manera que, para la planificación urbana, la participación de la sociedad es clave a la hora de dar respuestas a las situaciones problema y a los cambios propios de los espacios, considerando que se apuesta a una planificación participativa, en donde se privilegia el ámbito local. Esta planificación participativa plantea que las comunidades se involucren en el proceso, para lo cual el manejo de la información y el papel del planificador son elementos primordiales. Se apoya la idea de Longo et al. (2003), quienes indican que este tipo de planificación conlleva procesos dialécticos, plurales y multifacéticos, que se traducen en el uso de metodologías participativas y lineamientos estratégicos de desarrollo vinculados con la toma de decisiones "públicas o colectivizadas". Esto recuerda los planteamientos de Ceballos (2009), cuando señala que existe una transformación mutua individuo-realidad, gobierno-sociedad, que responde a la dinámica en la que se desenvuelve la ciudad.

Cabe destacar que, para la transformación de la realidad y revertir la problemática ambiental, se deben seguir una serie de lineamientos, tales como: 1- La indagación mediante la recolección de información bibliográfica, de campo y cartográfica; la caracterización socioeconómica y físico natural. 2- La interpretación de las posibles amenazas de los humedales urbanos para el logro de un manejo y acondicionamiento adecuado y la zonificación de sus espacios, al mismo tiempo que se detectan las amenazas de los ecosistemas. 3- La implementación de maneras de planificación lo que tiene que ver con la elaboración de planes de manejo, utilizando estrategias dictaminadas por la Convención Ramsar (2012), referidas al manejo de humedales urbanos, como investigación participativa aplicada, educación, comunicación y participación, recuperación, protección y manejo sostenible y de gestión institucional.

Estas acciones deben estar referidas al ambiente urbano y la identidad, análisis del contexto, interrelación de los componentes ambientales, reconocimiento y valoración de los humedales: su problemática y potencialidades; la gestión comunitaria y los espacios de uso público, la salud humana y la contaminación ambiental, la recuperación de los espacios degradados y los espejos de agua, manejo de los residuos sólidos urbanos, la reforestación y ornato urbano y predial y, por último, el mantenimiento y reposición con propuestas alternativas individuales y grupales de solución a las situaciones encontradas.

En la relación entre los elementos seleccionados para el manejo y acondicionamiento de los humedales urbanos se debe considerar lo siguiente: la preservación de la identidad, el fortalecimiento de la ciudadanía y el fomento de la diversidad cultural y la particularidad; el uso racional de los recursos, mermando los espacios agregados ocupados, y el fomento de una densidad urbana moderada; la expansión del transporte público y su interconexión con las obras existentes y nuevas. De igual forma, al mencionar urbanismo implica la vigilancia y la interconexión de espacios verdes de calidad y fomentando la conservación de los espacios públicos, garantizando la concordia y el avance de la interacción social y funcional; conservación de puestos de trabajo existentes y crear otros nuevos; creación de asociaciones entre la comunidad y los sectores públicos y privados; participación en los procesos de aprendizaje permanente; ver la vida urbana en su contexto más amplio, es decir, lo concerniente a la simbioempatía de los humedales en el contexto urbano mediante estrategias de urbanismo sostenible.

PA RA D I G MA

S O G I O G R I T I C O

C O M P L E J O E M E R G N T E

La relación entre las diferentes disciplinas permite salir de la limitada visión de conocimiento, reconociendo las posibilidades de una investigación producida bajo diferentes conceptualizaciones y logrando con ello un mejor entendimiento de los humedales urbanos y de la aplicación del conocimiento para la toma de decisiones. Se buscó implementar el cambio ontológico necesario para la conceptualización y estudio de los humedales urbanos, por lo que se considera al ser humano integrado a la naturaleza, custodiando los sesgos de cada disciplina que tienden a separarlos (Alvarado \& García, 2008).

Vale decir que los humedales son sistemas en los que la sociedad y los componentes biofísicos no pueden ser analizados separadamente, sino como un todo integrado, caracterizado por complejas interacciones y estrechas interdependencias. Esto es particularmente necesario cuando la disponibilidad del recurso hídrico es determinante en la dinámica funcional del 
socioecosistema. De esta manera, tanto el diagnóstico de los problemas como la definición de un objetivo común responden a necesidades del socioecosistema, es decir, a cuestiones tanto socioculturales como biofísicas.

La finalidad de comprender al objeto de estudio de una manera amplia, diversa y compleja permite en forma simultánea el desarrollo de aprendizajes de cada uno de los participantes de la comunidad. Se orienta la construcción de saberes para la acción, a partir de considerar a los individuos y la sociedad como producto de una historia de coadaptación y coevolución con el ambiente, en la que el hombre fue modificando su entorno para satisfacer sus necesidades, pero también formó parte de tales modificaciones. De esta manera, los individuos y la sociedad fueron construyendo su hábitat en esa cuenca, dándole significado a los elementos (humedales) y eventos (precipitaciones) importantes para su bienestar y en virtud de ello asignando formas de relacionarse y organizar la vida en la comunidad.

Es importante conocer las voces de los propios actores sociales para revelar los aspectos tanto materiales como simbólicos del sistema y los posibles caminos de solución que ellos mismos vislumbraban de acuerdo con su propia experiencia. En el diagnóstico del caso de estudio con base en la recopilación y revisión de la información generada por diversas fuentes se recurre a un intercambio constante de experiencias y aprendizajes, tanto de los usuarios finales del conocimiento generado como del grupo de investigadores, para así delimitar el problema considerando los intereses y las necesidades de los involucrados, y de este modo generar conocimiento pertinente tanto para los usuarios como para los tomadores de decisiones.

Se debe considerar fundamental involucrar diversas ciencias, explicitar los valores, deberes, obligaciones y prohibiciones que regularían la conducta en todo el proceso, asegurando que todo el trabajo se oriente a apoyar a las comunidades con quienes se establece el vínculo, puesto que la idea es generar conocimiento orientado a la acción.

\section{E L O B J E T I V O}

I N T E G R D O R Q U E

C O N T I E N E T O TALIIA D E S Y P A R T E S

El objetivo integrador de totalidades y partes es que surja un nuevo enfoque, donde se debe lograr que la comunidad asentada en el entorno de los humedales conozca y realice nuevas prácticas de manejo de estos ecosistemas para frenar o mitigar su deterioro (Riechmann, 2008). De tal manera que la holarquía como temática ambiental, relacionada con la problemática de los humedales urbanos y la reducción de la superficie ocupada, ha sido igualmente un proceso sostenido en el tiempo y se asocia directamente con una disminución de la calidad ambiental de sus paisajes ecológicos.

Paradójicamente, en el caso de otras ciudades más pobladas y debido a la ausencia de planificación ecológica o de ordenamiento territorial, los planes de crecimiento urbano no se compatibilizan con los objetivos ambientales del desarrollo sostenible y, consecuentemente, se expresan en un deterioro gradual, sistemático y persistente de los paisajes naturales, en circunstancias que las ciudades requieren mayores servicios ambientales en la medida que crecen y se desarrollan.

Por lo tanto, la planificación ecológica y la evaluación ambiental necesitan de un seguimiento cuidadoso de la evolución experimentada por cada ecosistema en particular, para lo cual existen instituciones encargadas en el país, pero no se le da la importancia correspondiente. Si bien la pérdida de áreas de alta calidad ambiental ha sido un proceso que ha afectado al humedal, ha significado una reducción importante de su superficie. Desafortunadamente, esta situación ha ocasionado cierta degradación en el ecosistema de la zona y ha tenido que soportar una importante reducción de sus áreas y un generalizado deterioro de su calidad ambiental.

De acuerdo con lo expuesto se propone la visión holística entre estos ecosistemas y la acción favorable de la comunidad, es decir, estableciendo relaciones armónicas hombre-ambiente que generen un manejo adecuado de este espacio tan vulnerable mediante una adecuada planificación de su uso (Tobón \& Núñez, 2006).

\section{E N F O Q E I N T E G R L}

El enfoque integral y la temática abordada constituyen las interacciones entre las partes y el todo (Garrafa, 2004). De acuerdo con esto, las partes son los componentes de los ecosistemas y toda la unidad de estudio, el contexto, es decir, los humedales urbanos; analizar el deterioro como consecuencia de la presión urbana y de ejecución de prácticas inadecuadas por 
parte de la comunidad en el manejo de estos espacios. Por supuesto, esto conlleva a conocer las características socioeconómicas y físico naturales del área. De igual manera, se estudia la situación social que afecta a sus integrantes, con el propósito de cambiarla mediante la participación de sus actores.

En primer lugar, se representa el análisis juntamente con los propósitos, obteniendo así las causas de la problemática ambiental en los humedales urbanos, complementando la interrelación de diferentes disciplinas con la teoría fundamentada sistémica mediante la observación participativa con los actores sociales.

En segundo lugar, es lo referente al paradigma emergente subjetivo donde se ubican los actores sociales, expresando cómo hasta el momento han utilizado los espacios de los humedales, sus creencias y costumbres. Es decir, la dimensión interna del actor social.

En tercer lugar, se muestra la interacción entre los investigadores y los actores sociales, lo cual se denomina la dimensión interna en colectivo. Se refiere al paradigma sociocrítico e interpretativo -donde las partes expresan su visión integradora- en el que se trata de comprender la realidad social de los humedales urbanos, desde la práctica, incorporando la autorreflexión como componente fundamental. Es importante destacar que desde esta posición se desprenden una serie de consideraciones que orientan hacia el acercamiento a los problemas sociales, reconociendo el potencial de cambio que tienen los actores implicados.

Por último, se encuentran los resultados, su análisis mediante el método deductivo. Se construye el concepto, la teoría donde se establecen las causas del deterioro de los humedales urbanos, generando a su vez un nuevo conocimiento, obteniendo la manera más eficaz del manejo y acondicionamiento de los humedales urbanos, como espacios vulnerables a ciertas actividades humanas mediante la planificación y acción de los involucrados.

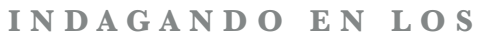 H U M E D A L E S U RA N O S}

En los últimos años se ha comenzado a tomar conciencia de la progresiva desaparición de los humedales urbanos, los cuales han estado sujetos a un descontrolado y continuo proceso de urbanización; se debe reconocer que no han sido suficientes las medidas adoptadas por los organismos competentes en sus diferentes niveles constitucionales de actuación, al no contar con normas, estrategias, políticas y otras herramientas, lo que facilita la apropiación de los recursos naturales por parte de actores públicos y privados en detrimento de mínimas condiciones ambientales y de seguridad.

Esto demuestra que no resultan suficientemente reconocidos por la población aledaña algunos de los principales atributos de los humedales urbanos que almacenan los recursos hídricos, actúan como reguladores de sus excesos y deficiencias, contribuyen a mantener la calidad de las aguas, sustentan una importante diversidad biológica, son hábitats para especies amenazadas, contribuyen a la estabilización del clima, son sitios de belleza paisajística y atractivos espacios para la recreación pasiva y contemplativa, para la investigación científica y la educación ambiental. Por esta razón, en la planificación para el manejo y conservación de los humedales urbanos se deben tomar en cuenta sus características físico-geográficas, tales como: precipitación, temperatura, evaporación, humedad relativa, vientos, insolación y radiación solar, uso consuntivo, geología, geomorfología, suelos, capacidad de uso de los suelos, hidrografía y drenaje, vegetación y uso actual y fauna.

De igual manera, hay que realizar el estudio correspondiente a sus atributos socioeconómicos de la comunidad aledaña a los humedales, siendo esta la población de interés, la cual está ubicada en los alrededores de los humedales donde sus patios son los frentes de dichos ecosistemas, mediante el abordaje de campo preliminar y los datos suministrados por ellos. Esto tiene que ver con predominio de población adulta o infantil, ingreso familiar, grado académico, ocupación u oficio, tipo de vivienda, servicios públicos, existencia de espacios recreativos, conocimiento de las funciones de los humedales, disposición de protegerlos, y las actividades conservacionistas que esta comunidad está dispuesta a ejecutar con el fin de poseer un lugar que les asegure el esparcimiento y la recreación para su propio bienestar.

Lo ideal sería que estas comunidades estén abiertas a un proceso de aprendizaje por medio de la implementación de charlas y talleres que conlleven a mejorar su calidad de vida en cuanto a los servicios que deben implementar los humedales urbanos se refiere, en primer lugar, a que cumpla las actividades propias de un espacio de esta naturaleza, así como actividades educativas y de investigación, no dejando a un lado las recreativas. 
Por otra parte, para obtener las causas de la problemática, denominado método deductivo desde una visión académica, hay que tomar en cuenta la forma en que deben relacionarse el desarrollo urbano y los humedales evitando su aproximación con uso de suelo habitacional, debido a la ausencia de respeto del ser humano por el medio ambiente, cuyas afectaciones con descargas o desechos, depredación de la flora y fauna, contaminación, invasión, elementos fundamentales para la subsistencia de las diversas formas de vida residentes en los ecosistemas frágiles. Para esto, los instrumentos normativos deberán estipular limitantes inquebrantables en su planeación, promoviendo una vigilancia que garantice la realización de lo establecido.

En la mayoría de los estudios de planificación se prioriza la exigencia de programas de educación ambiental en los diferentes ámbitos sociales, y se hace urgente que los entes gubernamentales ejecuten campañas de con- cientización ambiental que promuevan una cultura de respeto por los ecosistemas. De este modo, la educación en escuelas primarias y secundarias, estructuras educativas, políticas y sociales, deberían incluir programas con características clave para la acción y la participación ciudadana, con el objetivo de crear una cultura ambiental por medio de valores como la solidaridad, la cooperación, el respeto, el sentido de pertenencia e integración con el medio, entre otros, para crear futuros seres humanos que preserven su entorno.

Entre tanto, los espacios vulnerados son áreas frágiles desde el punto de vista ambiental, que son ocupadas por los seres humanos de manera irracional. La educación ambiental es el proceso educativo formal, no formal e informal que busca generar conciencia y cultura ambiental, así como la promoción de actitudes, aptitudes, valores y conocimientos, en beneficio del establecimiento de la sustentabilidad, según

\section{F I G U R A 4 .}

Parque Metropolitano Simón Bolívar. Cerros Orientales de Bogotá, Colombia. 28-07-20. Fuente: Felipe Casas R. Fotos y videos de Instagram (2021).

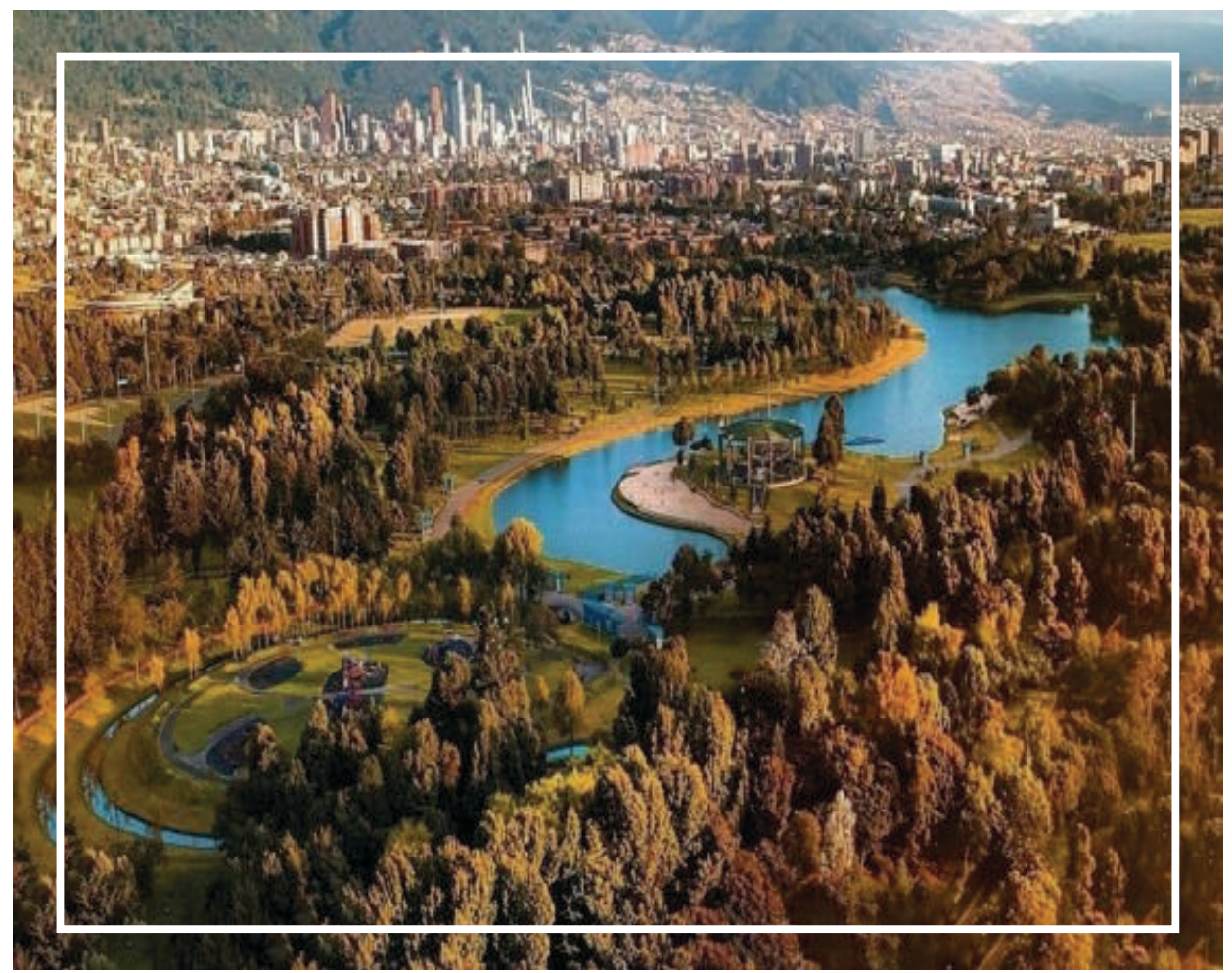


Meseguer et al. (2009). En lo que se refiere a normativa legal, como un conjunto de normas dictadas por un poder legítimo o una autoridad para regular la conducta o procedimiento que debe seguir un individuo u organización para cumplir con objetivos determinados. De esta forma, haciendo un contraste de definiciones se confirma el saber de los actores sociales, puesto que existen semejanzas en los conceptos mencionados, sin dejar de lado que se debe reforzar y actualizar este saber mediante un programa de capacitación de la población aledaña a los humedales generando conocimiento.

\section{S I T T E M A S S O G I A L E E N E L C O N T E X T O D E L O S H U M E D A L E S URBA NOS}

En todo este proceso se presenta la necesidad de estudiar varios aspectos relevantes de los humedales urbanos para entender su problemática y la urgencia de resolverla. Esto se relaciona con los sistemas sociales asociados a la planificación, políticas y gestión del desarrollo sustentable: postura crítica desde lo axiológico del manejo y acondicionamiento de estos ecosistemas y las principales causas y efectos del deterioro, siendo esto coyuntural para generar la simbioempatía en estos espacios frágiles como eje de articulación hacia una responsabilidad social compartida hombre-naturaleza.

Se presentan los sistemas sociales asociados con la problemática ambiental de los humedales urbanos, tomando en cuenta que el incontrolable crecimiento poblacional trae consigo el aumento sectorizado de la economía, avances de infraestructura y, al mismo tiempo, una paulatina reducción de las áreas naturales, lo cual pone en juego su sostenibilidad ambiental, teniendo en cuenta que un ambiente urbano es la relación sociedad-naturaleza luego de la transformación de espacios naturales, que genera un problema ambiental por exceso de carga de demandas de consumo social sobre el soporte territorial.

Se debe destacar que los problemas ambientales no son resultado del azar, sino del acontecer histórico social de una determinada comunidad humana interviniendo sobre su entorno para satisfacer sus necesidades materiales y espirituales. Además, esa relación sociedad-naturaleza, desde una visión totalizadora, o sea holística, está fuertemente influenciada por los aspectos sociales, económicos, institucionales, legales, políticos y culturales, todo esto mediado por los conocimientos científicos y las tecnologías aplicadas.

En la actualidad, el uso de la tierra, la introducción de especies y la interacción de estos aspectos con el cambio climático constituyen las variables de mayor impacto sobre los humedales, crea un claro punto de partida para la comprensión de la importancia de que los procesos hidrológicos y ecológicos, la diversidad biológica y los recursos naturales que suministran, que sean esenciales para el desarrollo y bienestar de los seres humanos, se traduce en la urgencia de una posición humanista para el manejo y acondicionamiento de los humedales urbanos.

Igualmente, se hace necesaria la identificación de los humedales, definiendo estos espacios hacia su aprovechamiento en el ambiente y desarrollo sostenible, caracterizando los niveles de satisfacción de estos en la población que los circunda, indicando los factores asociados a la satisfacción de la comunidad y conceptualizando las posibles estructuras donde se involucran los sistemas sociales, fundamentando cada uno de los elementos e interrelaciones para el aprovechamiento sustentable de la población que lo rodea.

Es imperioso tomar en consideración la intervención social, sanitaria, educativa, política, económica, ambiental, cultural, entre otros, más eficaces, que ayuden a conducir y regular las acciones individuales y colectivas para diseñar y poner en práctica modelos de acondicionamiento y manejo de los humedales urbanos. De igual manera, surge un intento de reformar la racionalidad sobre la que la ciencia y la tecnología se han venido apoyando. Esta actitud reformista afecta indistintamente tanto a las ciencias empírico-naturales como a las ciencias sociales y humanas y, en consecuencia, incide también en las ciencias de la educación. De aquí surgen los diferentes sistemas sociales, relacionados con la problemática ambiental de los humedales urbanos que a continuación se desglosan.

En cuanto al sistema político, vale decir que los problemas ambientales son conflictos políticos, es decir, problemas donde hay relaciones de poder. En otras palabras, en ellos se juega el resultado de dinámicas sociales que definen modelos de orden y participación humana, las cuales son determinantes en la construcción y transformación del territorio. La comprensión de la dimensión política de los problemas ambientales en los humedales urbanos se basa en el reconocimiento de que las relaciones 
políticas, que son relaciones de poder, son un elemento que constituye y transforma estos espacios, pues posibilitan eventos de habitar en los que se materializa la actuación de los seres humanos en el marco de los raciocinios culturales que les dan vida.

De allí que la figura de ambiente, entendido como el que privilegia la construcción de espacios habitados en los que se manifiesta la realización del ser, sea inherente al estudio de las relaciones de poder sobre los territorios, donde están involucrados los procesos políticos que se viabilizan en el marco y función de la obtención del manejo ideal de los humedales urbanos. Estos procesos políticos implican los derechos ambientales, los derechos humanos y las políticas públicas ambientales del Estado.

Por otro lado, al hablar de una nueva epistemología, que permita a la comunidad científica elaborar teorías más ajustadas de la realidad que posibiliten diseñar y poner en práctica modelos sociales, sanitarios, educativos, políticos, económicos, ambientales, culturales, entre otros, se habla de nuevos modelos teóricos que las diferentes ciencias deben adoptar, lo que le da el carácter de inter y multidisciplinariedad. De acuerdo con el sistema científico están involucradas las ciencias puras como la química, la biología, la física, entre otras; las ciencias sociales como la geografía, la ecología, y la investigación propiamente dicha, en el acondicionamiento y manejo de los humedales urbanos desde el punto de vista de la problemática ambiental que presenta.

Respectivamente, en el sistema jurídico de los ecosistemas frágiles, como los humedales urbanos, es de vital importancia implementar los estudios de impacto ambiental como procedimiento legal técnico administrativo para cada actividad que lo requiera. Esto es un ejemplo complejo de un sistema, que tiene por objeto la identificación, predicción e interpretación de los impactos ambientales que un proyecto o actividad produciría en caso de ser ejecutado, así como la prevención, corrección y valoración de los mismos. De acuerdo con esto, se deben cumplir una serie de normas y leyes ambientales que regulan el uso de los espacios en general y de los humedales en particular, generando el menor impacto negativo desde el punto de vista ambiental, donde la naturaleza es considerada como telón de fondo de la actividad humana y no como algo valioso jurídicamente por sí mismo.

Del mismo modo, existen tratados y convenios internacionales, como el Convenio
Ramsar (1971), para la protección de los humedales, la diversidad biológica, los recursos genéticos, los procesos ecológicos, los parques nacionales y demás áreas de vital importancia ecológica, velando por la existencia de un medio ambiente libre de contaminación en donde el aire, el agua, los suelos, el clima, la capa de ozono, las especies vivas, gocen de una especial protección. De allí, la existencia de leyes ambientales que integran los recursos naturales tradicionales y los elementos, factores y procesos ecológicos articulados a la biosfera y al propio ser humano, imponiendo la concepción política, filosófica y antropológica. Aunado a esto, la planificación y ordenación del territorio

Uno de los sistemas que legitima la acción de los gobiernos es el sistema económico, que básicamente trata de equiparar, por medio de recursos financieros, los costos ocasionados por el daño ambiental ocasionado. El brindarle un valor monetario a las acciones y efectos contaminantes permite generar un sistema de incentivos en el cual los entes generadores de contaminación eviten crear daños o, por lo menos, logren reducir de forma sustancial las acciones perjudiciales para el entorno natural.

Este sistema se expresa a partir de lo que la legislación ambiental en el mundo ha denominado tasas retributivas y compensatorias, que surgen a raíz de la utilización directa o indirecta de la atmósfera, del agua y del suelo, para introducir o arrojar desechos o desperdicios agrícolas, mineros o industriales, aguas negras o servidas de cualquier origen, gases y sustancias nocivas que sean resultado de actividades antrópicas o propiciadas por el ser humano, o actividades económicas o de servicio. También están las llamadas tasas por utilización de aguas, cuyo objeto es cobrar por la utilización de los recursos hídricos, con lo cual es posible la adquisición de dineros que cubran los gastos de protección y renovación de dicho recurso.

De esta forma, les corresponde a las autoridades ambientales destinar un porcentaje del recaudo de estas tasas a los procesos de protección y renovación de los cuerpos de agua ubicados en humedales, al igual que lo recaudado por aplicaciones penales de las leyes ambientales. Así pues, en los sistemas económicos se evalúan los indicadores relacionados con la huella hídrica, donde juegan un papel importante la economía ecológica y la economía ambiental, sobre todo en los humedales urbanos.

Entre tanto, el sistema de producción agrícola se relaciona con la problemática ambiental 
de los humedales, por la demanda de agua que esta actividad requiere, los impactos ambientales que ella ocasiona. De esta manera, se habla también de los sistemas agrarios y agroecosistema. En el caso de los humedales urbanos, en la actualidad, está el repunte de la agricultura urbana, que no es más que producir en los patios de las viviendas que conforman los frentes de estos humedales. Buena alternativa, donde los habitantes se ven obligados a no utilizar sus patios como basureros y pasan a ser patios productivos para su propia subsistencia.

Ahora es el momento de tocar el sistema religioso, tomando en consideración que la religión, como en toda sociedad es fundamental, puesto que involucra los valores, la fe, la creencia natural hacia lo sagrado. Así pues, la función de la religión como sistema social está conexa con la psicología: satisfacción de la necesidad de conocer. Está relacionada también con la deducción de conflictos, o sea la cohesión social, basada en la igualdad de los seres humanos, donde se justifican las decisiones políticas. Por último, la función ecológica de este sistema está referido a lo que contempla la Biblia en su libro de Génesis, donde habla de la creación del cielo y la tierra, el origen del universo que, al relacionarlo con la problemática ambiental de los humedales urbanos, tiene que ver con las diferentes sectas existentes, donde sus rituales se basan en el sacrificio de animales y el uso de sustancias nocivas y contaminantes que hacen desaparecer estos ecosistemas.

Es transcendental el tema de la ecología integral en el caso del sistema religioso, puesto que la Iglesia católica ha manifestado su preocupación por el problema ecológico relacionado con los problemas actuales de la sociedad. Las distintas Encíclicas papales, desde hace algún tiempo, se han interesado en expresar sus inquietudes, como lo comunicó Benedicto XVI en la Caritas in veritate que "cualquier menoscabo de la solidaridad y del civismo produce daños ambientales". También se pronunció ante la existencia de "una ecología del hombre, porque también el hombre posee una naturaleza que él debe respetar y que no puede manipular a su antojo".

Mientras que el Papa Francisco en el capítulo IV de la Encíclica Laudato Si (2015), presenta su inquietud diciendo que a la naturaleza no se puede separar del ser humano, es decir, los seres humanos son parte de ella. De acuerdo con esto afirma que "ya no es posible encontrar una respuesta específica e independiente para cada parte del problema. Es fundamental buscar soluciones integrales que consideren las interacciones de los sistemas naturales entre sí y con los sistemas sociales (...) hay una crisis socioambiental" (p.5). Muy claro está que el ser humano depende de la naturaleza para su propia existencia. De esto se desprende la necesidad de una ecología económica, donde se considere el humanismo que "convoca a los distintos saberes, también al económico, hacia una mirada más integral y más integradora". He aquí el término de economía integral, donde Laudato Si propone se sume la ecología cultural con el fin de salvaguardar las riquezas culturales en el mundo, puesto que su desaparición es tan grave como la desaparición de las especies.

En otro orden de ideas, se tiene el sistema mediático, relacionado con los medios de comunicación, puesto que los delitos ambientales y la problemática de los humedales urbanos no son cubiertos de manera completa y veraz. Aquí se destaca la cultura de las masas y el mundo de la publicidad, las esferas de la comunicación y la revolución digital, la retórica comunicacional y la globotomía ambiental. No se debe dejar de lado una de las formas de educación ambiental como es la informal, donde la utilización de los medios de comunicación es fundamental. Al igual que sus diferentes herramientas, como las tecnologías de comunicación, el sistema de información geográfica hace parte de ellas.

Seguidamente el sistema educativo; en este sistema se toman en cuenta los diferentes niveles de la educación y el aprendizaje significativo: aprender haciendo, que es de vital importancia en la problemática ambiental en general y en la de los humedales urbanos en particular, puesto que es la columna vertebral de todos los sistemas sociales y, por lo tanto, debe estar presente en todos y cada uno de los estudios donde de involucran diversas disciplinas.

Surge la educación ambiental como eje transversal en el sistema educativo, puesto que la concienciación sobre la solución de los problemas ambientales es transcendental para lograr el cambio de actitud hacia el uso de los recursos naturales por parte de los seres humanos, que conlleva a la relación armónica con el ambiente con miras a la sustentabilidad.

Por otra parte, es importante señalar el cambio de paradigma con respecto al concepto de familia; aquí surge el sistema familiar, puesto que se creía que era un núcleo o entidad aislada; en la actualidad esto ha cambiado, la familia es definida como la unidad de parentesco que mejor se adapta al sistema ocupacional 
económico moderno. Ante lo expuesto, el sistema familiar es la base para la solución de la problemática ambiental en los humedales urbanos, porque su consolidación hace que el uso de los recursos sea más fácil organizarlo y planificarlo y, por supuesto, el logro óptimo de la ordenación territorial.

Los problemas ambientales se pueden identificar con el abordaje de la relación entre ecosistemas y culturas, en el cual se producen alteraciones de los ecosistemas y su presencia es previa y constante al desarrollo de conflictos ambientales, que es una categoría concreta que involucra el accionar de los actores del conflicto en torno a la problemática. Así que la participación social en la resolución de conflictos ambientales no debe ser mecanicista ni el cumplimiento de un requisito normativo y/o institucional, sino que debe ir más allá, al contemplar los contextos particulares de las comunidades, las formas de organización, los valores y la forma de interacción con el entorno, las particularidades que existen en cada individuo, en cada familia, en cada grupo y en cada comunidad. En tal sentido es importante partir de conocer las poblaciones hacia las cuales se dirigen las acciones de organización comunitaria y las acciones institucionales. En esa medida, es pertinente realizar un acercamiento previo a las comunidades, caracterizar a la población, describir su contexto económico, político y social, que facilite y promueva la efectividad de la planificación de acciones a seguir y la solución del conflicto.

\section{A G I U D A D G O M O \\ S I S T E M A G OMPLE J O}

Así como se expuso en el punto anterior, sobre los sistemas sociales en los humedales urbanos, en este aparte se presentará la ciudad

F I G U R A 5 .

Biblioteca Virgilio Barco. Bogotá D.C., Colombia. 13-02-20.

Fuente: Felipe Casas R. Fotos y videos de Instagram (2021).

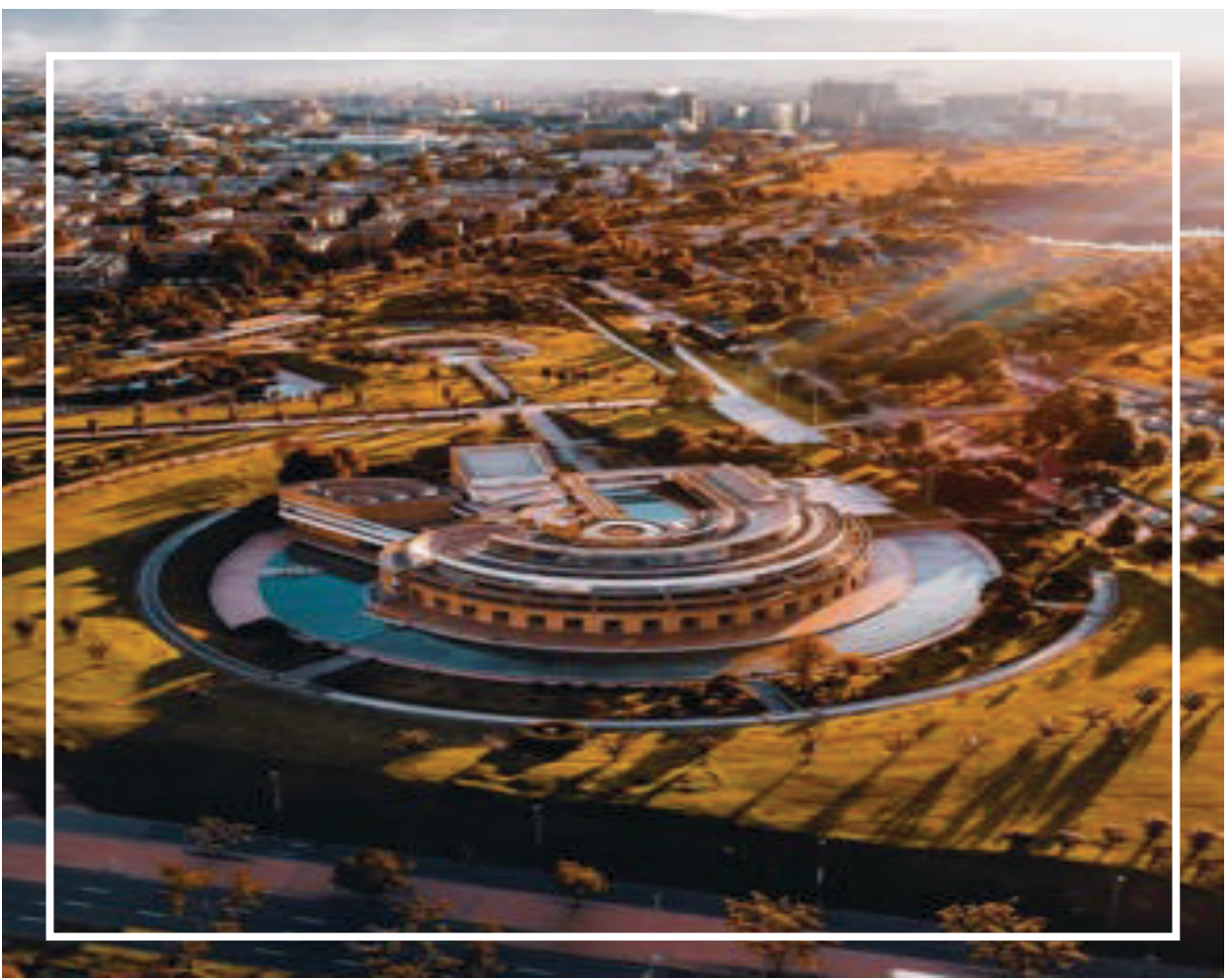


como sistema complejo, con el fin de entender su manejo como un sistema complejo o "el más complejo de los ecosistemas". En la ciudad, como en todo sistema, existen interacciones, interrelaciones e interconexiones complejas tanto entre los seres humanos como entre el resto de los seres vivos, y estos a su vez con su entorno, que determinan su sobrevivencia y/ supervivencia. Por lo tanto, lo ideal es que se genere de allí, relaciones de simbioempatía que permitan su permanencia en el tiempo y en el espacio que ocupan.

El progreso de los sistemas sociales está determinado por la resistencia a las perturbaciones del medio ambiente y, a su vez, están en adaptación continua con su entorno. Estas relaciones dinámicas, aunque complejas, permiten cierto nivel de independencia con capacidad de movimiento y de elección dando la oportunidad de organizarse. Precisamente de allí se desprenden una serie de subsistemas referidos a lo político, jurídico, económico, agrícola, religioso, mediático y familiar que actúan y tienen su origen de acuerdo con su cultura; y es la cultura la que permite la organización de las sociedades en instituciones que conllevan a la administración de las ciudades.

En nuestros días surge un nuevo subsistema, el científico-tecnológico, cuya principal tarea es la de alcanzar el bienestar de acuerdo con las innovaciones digitales en los aspectos políticos y económicos de las sociedades, que refuerzan la interconexión de las infraestructuras de la ciudad, originando una distribución de coexistencia regida por la "inteligencia artificial y los medios digitales", con tendencia a ofrecer mejoras en la calidad de vida de los ciudadanos. Todo este proceso de transformación se traduce en el nuevo concepto de Ciudades Inteligentes o Smart Cities.

\section{P LA N I F I G A G I Ó N, \\ P O L I T I C A S Y G E S T I Ó N D E L D E S A R R O L O \\ S U S T E N TA B LE : P O S T U R A G R I T I G A D E S D E L O A X I O L G I G O}

En los estudios ambientales convergen distintas disciplinas que van desde las ciencias biológicas, económicas, administrativas, hasta las sociológicas y jurídicas, entre otras, con diferentes planteamientos que, en general, se reducen al interés de cada materia en particular. Por otra parte, actualmente se cuenta con gran cantidad de investigaciones que han sido publicadas en diferentes medios. Igualmente, existe un sinnúmero de instituciones privadas y públicas, fundaciones, organizaciones no gubernamentales y centros de investigación que se preocupan por el aspecto ambiental. Sin embargo, se observa el fraccionamiento del conocimiento y una aplicación de políticas y programas de diferente índole en forma sectorial.

Lo anterior contrasta con la naturaleza misma del ambiente, en el sentido que está constituido por múltiples variables y elementos íntimamente relacionados que no pueden ser desligados unos de otros. Aunque se introducen nuevos conceptos y tecnologías que podrían ser muy útiles en la preservación, conservación y administración de los recursos naturales, aún no se cuenta con una metodología que permita gestionarlos de manera eficiente y eficaz. Por lo tanto, se presenta la importancia de la gerencia ambiental, cuyo propósito general es definir y desarrollar un sistema de gestión que incluya herramientas gerenciales idóneas para la administración de los recursos naturales y, por consiguiente, del ambiente.

De igual modo, en el acondicionamiento y manejo de los humedales urbanos se hace necesario tomar en cuenta que deben ser considerados zonas de mitigación del cambio climático y que se requiere conocer los supuestos teóricos que explican la existencia de los humedales urbanos, los tipos de estrategias para su manejo y recuperación, tomando en cuenta la definición de humedales, urbanismo, degradación ambiental y vulnerabilidad, los niveles de aceptación de estos espacios, los factores asociados en su aprovechamiento para conceptualizar una nueva visión de los humedales urbanos y su utilización, a fin de establecer la estructura conceptual y operacional de las políticas públicas seleccionadas para este modelo inherente al ambiente y desarrollo en el acondicionamiento y manejo bajo una posición racional.

\section{N D I G A D O R E S Q U E S E D E B E N T O MA R E N}

G U E N TA PARA FORMULAR PLA N E S, PROG RAMA S Y

P O L I T I C A S A M B I E N TA L E S E N L O H UM E D A E S UR B A N O S

Para la administración y gestión del ambiente es imprescindible contar con una estructura organizacional que facilite el flujo 
de recursos e información para apoyar la toma de decisiones que asegure el cumplimiento de las políticas ambientales. Esta información debería ser expresada en un sistema de indicadores del desarrollo sostenible que convendría ser integrado a los sistemas de indicadores sectoriales de otras instancias gubernamentales y privadas. Estos indicadores cobran vital importancia para el proceso de toma de decisiones, puesto que este requiere como condición un buen juicio y conocimiento de los problemas ambientales, además del uso de las diferentes herramientas gerenciales que soporten la acción en los diferentes roles y responsabilidades que les corresponda asumir a cada uno de los actores involucrados.

El proceso de selección de indicadores para varios países se ha venido realizando con el apoyo de las instituciones productoras y procesadoras de la información ambiental y con base en las observaciones y sugerencias de los grupos de trabajo, tanto de la Comisión Económica para América Latina y el Caribe, CEPAL, como de la Asistencia Técnica de Cooperación Horizontal de la Autoridad Nacional del Ambiente de Panamá (ANAM). Cabe destacar que la asesoría internacional fue de gran importancia para el desarrollo de este grupo de indicadores ambientales.

Para el tema a tratar, referido a los humedales urbanos, debido a la complejidad de estos ecosistemas y su alta fragilidad ambiental, se deben tomar en cuenta todos los indicadores ambientales que tengan que ver con atmósfera, bosques, diversidad biológica, energía y transporte, gestión ambiental, residuos y desechos sólidos urbanos, tomando en cuenta, también, que estas áreas han sido sometidas a cambios bruscos debido a la alta intervención antrópica. Por lo tanto, es imprescindible contar con una estructura organizacional que proporcione los recursos e información para apoyar la toma de decisiones que asegure el cumplimiento de las políticas ambientales. Esta información debería ser expresada en un sistema de indicadores del desarrollo sostenible que debería ser integrado a los sistemas de indicadores sectoriales de otras instancias públicas y privadas.

Estos indicadores cobran vital importancia para el proceso de toma de decisiones, ya que este requiere como condición, un buen juicio y conocimiento de los problemas ambientales, además del uso de las diferentes herramientas gerenciales que soporten la acción en los diferentes roles y responsabilidades que les corresponda asumir a cada uno de los actores involucrados. Es de destacar que deberían ser más específicos para cada caso en particular y no tan generales, como el caso preciso que compete a los humedales urbanos.

\section{A P L A N I F I G A G I Ó N D E} P O L I T I G A S A M I E N T A E S RELAGIONA DA S G O N LOS H U M E D A L E S U R B A NOS

La política ambiental se ha caracterizado por la aplicación de controles directos y, como consecuencia, la gestión de la contaminación y los recursos naturales se apoya en el cumplimiento de una extensa y compleja legislación. Por ello es necesario la elaboración de la declaración de política ambiental para ordenar las acciones actuales en materia de gestión ambiental.

Es lamentable que aún en la actualidad los problemas ambientales más importantes no sean atendidos de manera adecuada. Tal es el caso de la degradación que presenta en estos momentos el ambiente, consecuencia de la expansión comercial y la industrialización de las empresas en todo el planeta, puesto que a la mayoría de estas no les importa lo que pueda pasar con el medio de donde adquieren los recursos para la producción de sus bienes, mucho menos cómo renovarlo, pues lo primordial para ellos es la obtención de beneficios propios.

Una de las industrias que más impactos ambientales genera a nivel local y global es la petrolera y sus distintas fases de su explotación y minera en general, las cuales generan destrucción de la biodiversidad y del ambiente; y los impactos en las poblaciones locales que viven alrededor de la infraestructura petrolera y minera se evidencian incluso antes de que se inicie cualquier operación, tanto así que cientos de comunidades han sido desplazadas alrededor del mundo para dar paso a esta industria, y cuando la gente tiene que convivir con ella enfrenta serios problemas de salud, de pérdida cultural y de violencia.

Es entonces por lo que se denota un desequilibrio en el crecimiento no solo económico sino también demográfico, donde se ve con claridad problemas sociales, educativos, poblaciones cada vez más empobrecidas, crecientes cordones de marginalidad, colapso de los servicios públicos, ecosistemas agotados, que afectan directamente la calidad de vida de la población propia del área, así como también aquellos que migran en busca de supuestas mejoras. De esto no escapan los humedales en todo el mundo, convirtiéndose en humedales urbanos sin 
ningún tipo de medidas para su protección y conservación, ni un manejo eficaz que permita la relación armónica del ser humano y estos importantes ecosistemas.

Venezuela, por ejemplo, posee una importante riqueza paisajística, climas variados y un potencial turístico inmenso, por lo que es inaceptable que ni siquiera se evidencie la voluntad de reparar los daños ambientales causados por las empresas estatales y no se tome con seriedad el cambio climático ni la educación ambiental. Se observa cómo la gestión ambiental se ha reducido a acciones aisladas que solo buscan resolver un problema puntual, como la construcción de infraestructuras de agua potable, aguas servidas y desechos sólidos, etapas que debieron ya ser superadas.

Todos estos esfuerzos se malgastan cuando no existe una política ambiental que abarque la realidad del país. Si bien hay que valorar las iniciativas legislativas y constitucionales evidentes, no se considera que existen unos principios regentes en materia de gerencia ambiental que incluyan conceptos como la protección, conservación, pero sobre todo preservación del ambiente en los humedales. Por todo esto y más, es necesario retomar la ordenación del territorio, promover el desarrollo sustentable y fortalecer al Estado para asumir la rectoría y control de los procesos que traen consigo el progreso; es aquí donde las políticas públicas en materia ambiental -que son el conjunto de acciones emanadas de los poderes públicos con el fin de minimizar los comportamientos contaminantes o depredadores de las personas que habitan dentro de la jurisdicción de esos poderes públicos- tienen el sustento legal como los tratados internacionales y demás leyes ambientales de planificación y gestión de la ordenación del territorio, entre otras.

A raíz del creciente interés generado por la problemática ambiental a nivel mundial, los gobiernos se han visto presionados a desarrollar políticas y estrategias ambientales que tengan como objetivo lograr el control de la contaminación ambiental y el manejo de los recursos naturales, al menor costo social posible. Para lograr este objetivo, los gobiernos deben desarrollar políticas ambientales coherentes que incluyen como paso fundamental la selección, el diseño e implantación de instrumentos económicos o de mercado para lograr alcanzar las metas propuestas. Entre tanto, la problemática ambiental es el resultado del modo por el cual la sociedad se ha organizado en el territorio y ha hecho uso del ambiente, para satisfacer sus necesidades de producción, distribución y consumo de bienes y servicios.

Por otra parte, a pesar de los adelantos tecnológicos y avances científicos, los desechos continúan siendo uno de los problemas sanitarios más importantes dentro de nuestras comunidades, sobre todo en aquellos lugares que por sus características de desarrollo tienen sus habitantes concentrados en los centros urbanos no planificados, donde los servicios públicos suelen ser deficientes.

Para ello se propone realizar campañas de concientización a todas las comunidades, programas educativos ambientales, avisos y recolectores de basura con su distintivo del tipo de desecho, disminución de impuestos, brigadas ecológicas, campañas para divulgación de la gama de leyes existentes, entre otras; una vez que el gobierno haya implementado todas esas medidas se deben aplicar sanciones correspondientes a los ciudadanos que violen la norma. Por otro lado, que sea requisito necesario que para poder concursar a un cargo público como presidente, gobernador, alcalde, concejal, debe por lo menos poseer conocimiento previo en materia ambiental, para que así pueda tenerlo en cuenta a la hora de planificar lo ambiental.

Muchos son los países que carecen de leyes que regulen la administración y cuidados de sus espacios naturales, o las que tienen no son suficientes para garantizar esta difícil tarea; en otros son abundantes, sin embargo, hay que profundizar su cumplimiento mediante la acción colectiva y tener en cuenta la nueva perspectiva respecto a la búsqueda de un ambiente sano, seguro y ecológicamente equilibrado, que a través de la aplicación de las leyes puede fortalecerse con la articulación, donde hay cierta debilidad. Esta articulación no se ha dado de manera efectiva, y aun cuando se tenga la misma visión estratégica esta tiene que irse desagregando, pero con la misma direccionalidad, para que quienes puedan actuar lo hagan conforme a sus competencias.

Retomando los humedales urbanos, se cuenta con acuerdos administrativos y legales que responden a la protección y al manejo de cada ecosistema, así como la existencia de las bases legales adecuadas, las cuales son elementos fundamentales para el éxito a largo plazo de estos ecosistemas y están enmarcadas dentro de los parámetros de la administración, manejo y apoyo para actuar en cualquier momento. En el ámbito internacional, en 1971 se adoptó la Convención Ramsar, "Convención relativa a 
los humedales de importancia internacional, especialmente como hábitat de aves acuáticas". También el Convenio sobre diversidad biológica de 1992, como marco de referencia para la generación y el desarrollo político y normativo con respecto a la conservación y protección de ecosistemas estratégicos. En este último se busca la conservación de especies mediante la adopción de medidas como la creación del sistema de áreas protegidas y otros mecanismos especiales de protección.

I M P L I C A G I O N E S D E LA FORMULAGIÓN DE P O L I T I A S A M I E N TALES DESDE LA VISIÓN PARA R E V E R T I R E L G A M B I O G L I M Á T I G O Y L O S H U M E D A L E S URBANOS

Las amenazas de desintegración sistémica derivadas del cambio climático combinado con la economía no parecen ocupar hoy un lugar preponderante en la agenda pública, al tiempo que no se logran articular soluciones colectivas ni convocar actores capaces de llevarlas a cabo. Los estudios disponibles sobre el tema indican que no solo los efectos del cambio climático tienen impactos distributivamente regresivos, sino que además las políticas que apuntan a mitigar esos efectos pueden también tener impactos negativos en ausencia de otras políticas. Las excepciones son pocas y se reducen a aquellas políticas que apuntan al transporte personal que suelen indicarse como más crecientes.

Los resultados dependen de las técnicas aplicadas en los estudios y del modo en que se consideran los comportamientos de los funcionarios en reacción a las políticas. Estos problemas se expresan de un modo característico en América Latina, donde la diversidad de los países y los problemas estructurales de desigualdad no son un campo favorable para el discurso que cuestiona los daños ambientales del régimen económico actual. Mucho menos en un contexto de crisis internacional, del cual algunos países de la región parecen beneficiarse con un período de crecimiento inusual y mejora de indicadores sociales. Por otra parte, en la región el problema no se circunscribe a las emisiones de carbono de ciertas industrias, sino que abarca otras cuestiones vinculadas al daño ambiental como la deforestación, la minería a cielo abierto, la contaminación de reservas acuíferas, la extracción de recursos gasíferos con métodos no convencionales, el agotamien- to de suelos por uso de agroquímicos, entre otros.

Así que el modelo de crecimiento que en la actualidad amenaza el medio ambiente se vincula tanto con ciertas tecnologías de industrialización como con el modelo de crecimiento extractivo de recursos naturales en muchos países. Un crecimiento económico con un contenido más verde coloca nuevos desafíos tanto al sistema de acumulación como a los sistemas de protección social, empezando por la baja densidad de creación de empleo y la necesidad de integrar múltiples políticas dadas las múltiples dimensiones de la vulnerabilidad social. A los ya complejos riesgos sociales que enfrenta la región, el cambio climático agrega nuevas demandas para disminuir el consumo notable y posicional, genera requisitos fiscales adicionales, tanto por el lado de los ingresos como de los gastos, y sobre todo plantea nuevos dilemas distributivos.

Por lo tanto, la controversia de los sistemas de producción y consumo debería hacerse teniendo en cuenta la necesidad de pasar a un régimen de crecimiento moderado, pero más estable y armónico. Al mismo tiempo, se vuelve prioritaria la defensa de consumos básicos, incluyendo a los servicios sociales. La cuestión distributiva se coloca así como una dimensión esencial del problema.

Hoy en día, los países latinoamericanos muestran diferente capacidad institucional para enfrentar estos desafíos. En términos generales, en la mayoría de los países se vuelve central revisar: la visión sobre el conflicto temporal entre el corto y largo plazo en economías muy volátiles y con tendencia recurrente a sufrir crisis; la identificación y discusión de estándares básicos que es necesario alcanzar, pero además de aquellos que hay que conservar y defender; la centralidad del empleo como forma única de acceso al ingreso y como forma principal de acceso a elementos básicos para el bienestar (salud, pensiones); la forma de evaluación del progreso social y de los indicadores de privación y opulencia. Estas políticas necesitan ser legitimadas.

Igualmente, el cambio climático debe integrarse como un nuevo riesgo social estructural que deben atender los sistemas de protección social y que potencia la recomendación de reformar sus instituciones. Si bien el uso de los fondos captados por los nuevos impuestos verdes puede servir para atender los problemas que han de crear las propias políticas de mitigación del cambio climático, esto no es 
suficiente. Son necesarios cambios estructurales en la organización de los sistemas sociales. Para ello, aquí se sugiere pensar en las instituciones que deberían integrarse para atender estos problemas organizados en cuatro grupos distributivos: emisiones de carbono; empleo/ tiempo; consumo/inversión e ingreso/riqueza.

Este tipo de desafío no parece posible en las actuales instituciones inerciales de los sistemas de protección social en la región. La intuición es que aquellos modelos con esquemas más universales de redistribución y sistemas económicos coordinados estarán en mejores condiciones para adaptarse a los llamados estados de bienestar. Este criterio debe servir para orientar el rediseño institucional. En este rediseño, las nuevas políticas atentas a los desafíos del cambio climático deberían: integrar los objetivos ambientales sostenibles en objetivos básicos de la política social; incorporar las incertidumbres y complejidades asociadas con el cambio climático en el análisis de las políticas sociales; asegurar que las políticas económicas estén diseñadas para satisfacer el núcleo social y ambiental, y no simplemente los objetivos de crecimiento; crear empleo y facilitar la adopción de empleos de la economía verde en los grupos desfavorecidos y reducir las desigualdades y desventajas arraigadas por medio de políticas sociales.

De acuerdo con lo expuesto y tomando en cuenta que los humedales son ecosistemas vulnerables que juegan un papel fundamental en la dinámica hidrológica, cultural, ecológica y socioeconómica, que siguen deteriorándose por causas asociadas al cambio climático y a la presión generada por actividades antrópicas, su conservación ha despertado el interés en la búsqueda de elementos conceptuales, herramientas de evaluación y alternativas de gestión que permitan direccionar de mejor manera el manejo de estos ecosistemas mediante nuevas visiones y comprensiones en torno a la mitigación y adaptación. Por lo tanto, se debe hacer una revisión en torno a los procesos de degradación de los humedales urbanos, exponer la vulnerabilidad y resaltar el reto actual de desarrollar los análisis por medio de una visión que integre lo social y lo biofísico, generando un modelo teórico para su acondicionamiento y manejo adecuado, destacando a la vez que estos espacios se pueden constituir en unos verdaderos centros de educación de participación ciudadana.

\section{LA G E S T I Ó N A M B I E N TAL S U S T E N T A B L E D E L O S H U M E D A L E S U R B A N O S}

Desde una visión teórica se hace necesario rescatar la importancia de la gestión ambiental como manejo de las actividades o conductas del hombre, que influyen de manera relevante sobre el ambiente, haciendo énfasis hacia la protección ambiental; no debe entenderse como función exclusiva del Estado, razón por la cual el derecho a la información y a la participación ciudadana es constitutivo del derecho a un entorno sano. A ello se le engloba el hecho de los ordenamientos jurídicos, donde se aprecia homogeneidad en cuanto a la consagración y desarrollo del derecho humano a un ambiente sano mediante la incorporación en los textos constitucionales y en leyes ambientales, políticas públicas que incluyen pautas, principios y políticas de carácter general, lográndose así armonizar la protección del ecosistema con el contexto socioeconómico y cultural de la población (sustentabilidad), para que a la par la educación verde se haga más aplicable y efectiva hacia la ejecución de las normas jurídicas ambientales. En este sentido, el derecho ambiental como fuente e instrumento de la política de esta estimularía la función ciudadana de la gestión propiamente desarrollada.

Por otra parte, todo desarrollo ecológico debe ser dirigido hasta las esferas superiores de cualquier organización para fundar en ella la necesidad de conciencia sobre las acciones ambientales, sin fallas a leyes o doctrinas que rijan el tema, evitándose así que estos se involucren en delitos penales por decreto ambiental. Por lo tanto, la sustentabilidad viene enfocada mediante la base epistémica del desarrollo sustentable, es el equilibrio entre lo ecológico, lo social y lo económico, es la garantía de que las generaciones presentes puedan satisfacer sus necesidades sin comprometer las posibilidades de las generaciones futuras.

Igualmente, la percepción y el conocimiento de las personas sobre los problemas ambientales suelen ser tangenciales, haciendo uso de los recursos naturales sin ser verdaderamente conscientes de ello, debido al hecho de ser conductas y actitudes que en conjunto conforman el concepto de conciencia, siendo esta la que contribuye directamente hacia la formación integral de la persona, dentro de su educación como acción a todos los niveles.

De ello que la educación para la sustentabilidad pretenda ser ese eslabón activador de la conciencia ecológica en la persona. Este tér- 
mino es definido como el método de vivencias, conocimientos y experiencias que el individuo utiliza activamente en su relación con el medio ambiente. Es un concepto multidimensional en el que han de identificarse varios indicadores, de los cuales concretamente se pueden distinguir cuatro dimensiones:

- Cognitiva: referida al grado de información y conocimiento sobre cuestiones relacionadas con el medio ambiente. Se habla en esta dimensión de IDEAS.

- Afectiva: enfocada a la percepción del medio ambiente, creencias y sentimientos en materia medioambiental, se refiere a las EMOCIONES.

- Conativa: dirigida a la disposición para adoptar criterios en pro del medio ambiente en la conducta, manifestando interés o predisposición a participar en actividades y aportar mejoras. En esta cualidad se evalúan ACTITUDES.

- Activa: es la realización de prácticas y comportamientos ambientalmente responsables, tanto individuales como en conjunto, inclusive en situaciones comprometidas $\mathrm{O}$ de presión; se estudian CONDUCTAS.

Para que un individuo adquiera un compromiso con el desarrollo sustentable que integre la variable ambiental como valor en su toma de decisiones diarias, es necesario que alcance un grado adecuado de conciencia ecológica a partir de unos niveles mínimos en sus dimensiones cognitiva, afectiva, activa y conativa, dejando en claro la acción de estos niveles en forma de sinergia, así como dependientes del ámbito geográfico, social, económico, cultural o educativo en el cual el individuo se posiciona.

Finalmente, toda acción dentro de la educación ambiental es facilitar la resolución de un determinado problema ecológico. La conciencia ambiental o ecológica determina las decisiones en este ámbito, por lo que analizarla, diagnosticarla y desarrollar herramientas para potenciarla constituye un paso básico a la hora de diseñar y realizar planes y programas eficientes de materia de políticas de Estado que desean regular.

En otro orden de ideas, la política ambiental se caracteriza por la aplicación de controles directos, por lo que cualquier experiencia en instrumentos económicos es escasa y aislada, el nacimiento de una consecuencia abierta, la desmotivación al cumplimiento con el ambiente. De tal manera que la gestión de los recursos naturales, o gestión ambiental, en el caso especial de los humedales urbanos, se debe apoyar en el cumplimiento tanto de una extensa legislación como por su apertura ante las sanciones sobre fallos ambientales; sin embargo, la contabilización tanto de los costos ambientales como de políticas de fijación de precios de los recursos naturales no han sido desarrollados dentro del actual sistema de mercado, desconocimiento y no cumplimiento por vocación dentro de las organizaciones. Por lo tanto, se deben regular los usos de estos espacios para disminuir su afectación que conlleva a la reducción de su superficie hasta su desaparición, inclusive lograr la sustentabilidad.

Los graves problemas ambientales que se registran en la actualidad cada vez son mayores $\mathrm{y}$ en crescendo, conllevando al calentamiento del planeta, convirtiéndose en el llamado cambio climático, deben ser objeto de una actuación integral, por parte no solo de los ciudadanos de cualquier país, sino también de todas las organizaciones que lo conforman en su contexto económico, social, cultural y político. Cada vez más se identifican en la sociedad personas, grupos sociales y organizaciones, las cuales manifiestan con sus acciones proambientalistas un grado de conciencia ambiental que ayude, desde diversas perspectivas, a dar impulso a las mejores soluciones posibles para dicha problemática.

Tomando en cuenta que todo lo expuesto se resume en la gestión ambiental sustentable con el propósito de disminuir, mitigar y controlar la problemática ambiental existente en los diferentes espacios utilizados u ocupados por el ser humano, no escapa de ello la ocupación de los humedales, llamados urbanos por estar rodeados de asentamientos poblacionales, por lo cual requiere de acondicionamiento y manejo con una posición humanista o, lo que es lo mismo para el caso, una posición racional. En tanto que se deben tomar algunas medidas simples como que el ciudadano común debe crear internamente un despertar por la equidad y el 
cuidado de las áreas de esparcimiento urbano y rural, que no se espere hasta tener la sanción para desarrollar una acción específica que lo haga partícipe del ecosistema como valor agregado en él y no como factor destructor.

$\mathrm{Al}$ igual que a los medios de divulgación de información, es necesario crear líneas de promoción donde se profese en el ciudadano, el empresario, el rector de gobierno y en todos los despertares de la conciencia, evitando daños al ambiente más por convicción que por miedo a la penalidad. Estos medios llegan a todos los niveles sociales y deben actuar de la mano de entes de gestión de políticas ambientales y educación.

\section{P R I N G I PA L E S GA U S A S Y E F E C T O S D E L D E T ER I O R O D E L O S} H U M E D A E S URAANOS

De acuerdo con las principales causas y efectos del deterioro de los humedales urbanos, se deben considerar los siguientes aspectos:

1. Mejoramiento de la planificación del desarrollo urbanístico de la zona: la falta de planificación territorial ha sido una de las carencias culturales e históricas, esto debido al incumplimiento de las políticas de ordenanza municipal, lo cual ha conllevado a situacionescríticasentodasuextensión.

La progresiva proliferación de ranchos, causado por las invasiones, ha ocasionado la creciente disminución de los espacios naturales, lo cual ha traído como consecuencia la migración de ciertas especies de fauna y la alta disminución de especies forestales. Es necesario e indispensable evaluar los impactos ambientales en cualquier zona donde se pretenda desarrollar e intervenir un área. Por eso, se les sugiere a los organismos nacionales, estatales y municipales cumplir con las legislaciones ambientales y ordenanzas territoriales correspondientes.
2. Afectación de la biodiversidad: el impacto creciente de las actividades diarias en la zona de los humedales puede generar una pérdida de biodiversidad acelerada. La causa principal es la destrucción de ecosistemas de gran interés, tala forestal, quema incontrolada de maleza, cuando cambian la calidad del agua o la atmósfera por la contaminación, lluvias ácidas por efecto de agroquímicos o emisiones atmosféricas. Todo esto aumenta la deserción y extinción de la flora y fauna. Por lo tanto, se requiere de una concientización ambiental en todos los ámbitos sociales y culturales, así como también el cumplimiento de las leyes ambientales.

3. Respecto a la reducción de los humedales: la afectación resulta un acto antijurídico, ambientalmente hablando, y antiecológico, debe evitarse que continúe ocurriendo, porque los efectos generados a estos ecosistemas son irreversibles, además que se lesiona la biodiversidad que allí hace presencia, se afecta además el entorno, afecta el planeta. La flora y la fauna acuática y las aves, al ver reducido su espacio natural se obligan a migrar a otros lugares, además que sienten más cerca la presencia de los pobladores que de forma drástica han ido sustrayendo gran terreno de los humedales para urbanismos.

Como consecuencia de lo planteado, en época de lluvias va a ocasionar mayores inundaciones en el sector, pues las aguas que solían distribuirse en mayor superficie hoy cuentan con menor área para ser recibidas, y si no se construyen los canales de desagüe necesarios, se producirán inundaciones en los nuevos asentamientos. Dicho lo anterior, las autoridades ambientales competentes y la administración pública deben 
avocarse a hacer cumplir las normas establecidas para la protección de estos espacios naturales.

4. Depreciación de la calidad de vida de los habitantes: es una realidad que los habitantes de los humedales, por carecer de una justa planificación de construcción de viviendas, en un área adecuada, ven en desmedro su calidad de vida, pues además de las plagas con las que tienen que convivir, su salud se encuentra seriamente afectada, porque la realidad es que conviven con cría permanente del mosquito Aedes aegypti, transmisor de del virus del dengue, de la fiebre amarilla, entre otras.

Por lo tanto, debe hacerse más férreo el cumplimiento de las normas ambientales, aplicar un estamento jurídico, sea el universal suscrito por el Estado o el interno, porque solo con su estricto cumplimiento no solo se protegerá el humedal, sino que el mayor beneficiario será el planeta y las generaciones por venir recibirán este aporte.

Por lo expuesto, las principales causas y efectos del deterioro de los humedales urbanos han ocasionado en estos ecosistemas la pérdida de área y descomposición, modificación del régimen hídrico, presencia de especies invasoras, depreciación de hábitats de fauna y destrucción

F I G U R A 6 .

\section{Árbol del problema.}

Fuente: elaboración propia (2021).

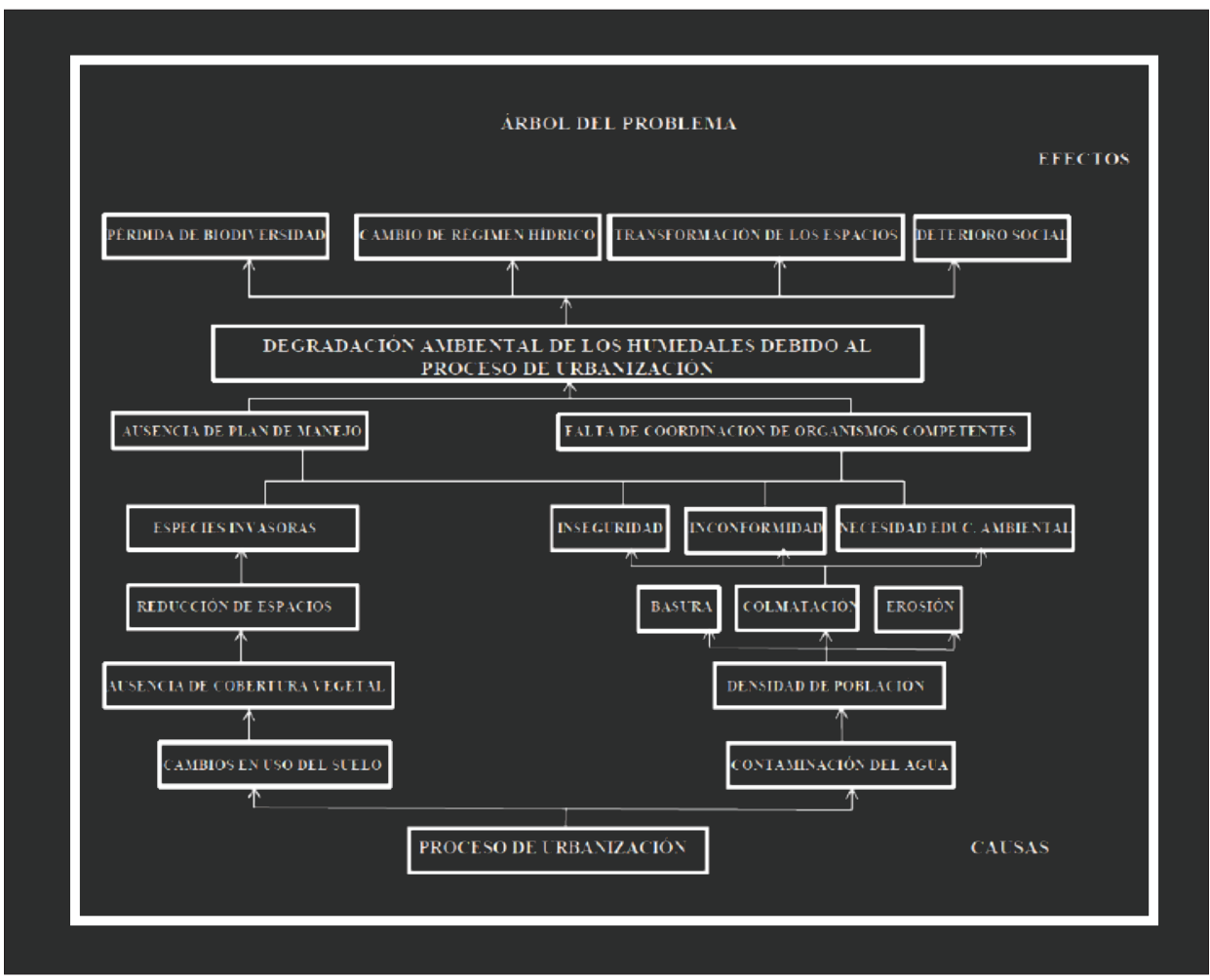


de vegetación autóctona, entre otros fenómenos negativos. De esta manera, se estableció un árbol del problema mediante la entrevista con los actores sociales, donde se representan las causas y efectos de la mencionada degradación de estos ecosistemas. En la siguiente figura se puede observar en resumidas cuentas lo expresado en los párrafos anteriores.

F I G U R A 7 .

Aspectos epistemológicos en humedales urbanos..

Fuente: elaboración propia (2021).

\section{Epistemología}
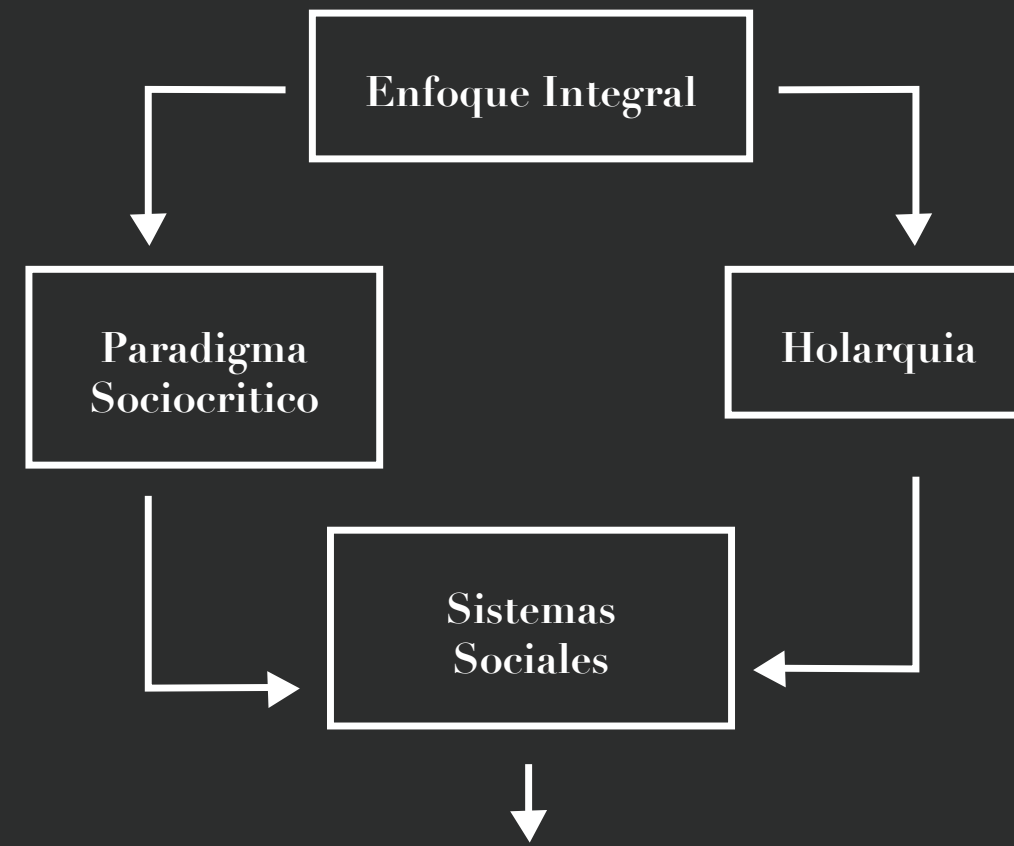

Planificacíon y gestion ambiental en humedales urbanos 